\title{
Exfoliated Graphite reinforced Acrylonitrile butadiene styrene Composites
}

\author{
K B Bisal ${ }^{1}$ and Kamal K. Kar ${ }^{1,2^{*}}$ \\ ${ }^{1}$ Advanced Nanoengineering Materials laboratory, Materials Science Programme, Indian Institute of \\ Technology Kanpur, Kanpur-208016, India \\ ${ }^{2}$ Advanced Nanoengineering Materials laboratory, Department of Mechanical Engineering, Indian \\ Institute of Technology Kanpur, Kanpur-208016, India, \\ *Email:kamalkk@iitk.ac.in
}

Exfoliated graphite (EG) as a filler material in advanced nanocomposites has achieved great interest in the recent past. This is due to its unique properties such as large surface area, low density, low cost, good electrical conductivity, etc. The EG/polymer composites are found applications in high temperature gaskets, electronic packing, electrodes, etc. The main objective of the present study is to synthesize EG/acrylonitrile butadiene styrene (EG/ABS) nanocomposite and to study the thermal conductivity variation as a function of EG loading within the nanocomposite.

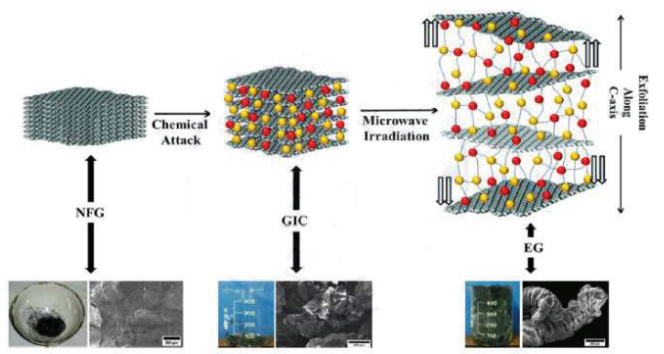

Figure 1: Schematic of exfoliation of graphite

Initially, EG is synthesized from natural flake graphite (NFG) precursor by microwave irradiation technique. NFG is mixed with intercalating and oxidizing agents such as, $\mathrm{HClO}_{4}, \mathrm{HNO}_{3}$ and $\mathrm{KMnO}_{4}$ leads to the formation of graphite intercalation compound (GIC). It is followed by microwave irradiation, which leads to the expansion of graphite layers along $c$-axis. The schematic showing the process of synthesizing EG is shown in Figure 1. The density of EG is $0.055 \mathrm{~g} / \mathrm{cm}^{3}$. Further, EG/ABS nanocomposite is prepared by injection moulding with the help of micro compounder. Four EG/ABS nanocomposite nanocomposites are prepared by varying the EG concentration (in vol \%) as 20,30, 40 and 50 .

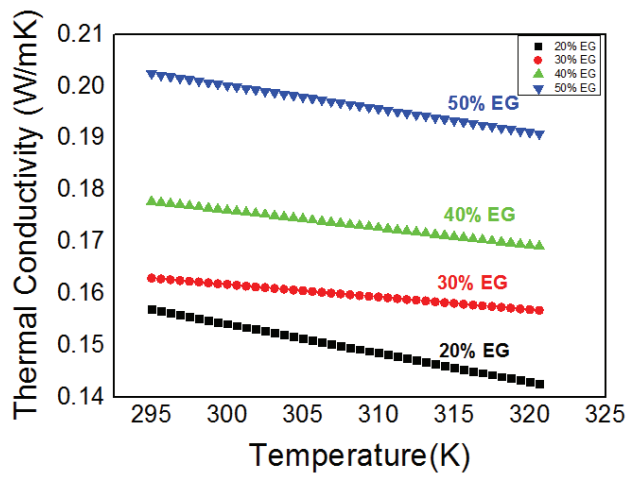

Figure 2: Graph of thermal conductivity of EG/ABS nanocomposites at different temperatures

Thermal conductivity measurment of composites has been performed using laser flashmethod. Thermal conductivity of the EG/ABS nanocomposites is found to increase upon EG loading in the EG/ABS nanocomposite and the variation is shown in Figure 2. An increase in the thermal conductivity can be observed at high EG loading and it may be attributed to increase in integrated auto correct of net power exchange, which is propotional to dencity of composite. The incorperation of EG makes conductive pathways, which increases thermal conductivity of the nanocomposite.However thermal conductivity decreases with temperature due to scattering of phonons.

\section{References}

1. Sengupta Rajatendu, Bhattacharya Mithun. Polym. Sci., 2011, v. 36, pp. 638-670.

2. Rajeev K. Gautam, Kamal K Kar, Advanced Science, Engineering and Medicine,7,4,(2015) 429434.

3. A. Shenavar, F. Abbasi Research Center for Polymeric Materials, Sahand University of Technology, Tabriz, Iran. 